\title{
Concentración y crecimiento en Chile: una relación negativa ignorada
}

Miguel Atienza. Instituto de Economía Aplicada Regional (IDEAR), Universidad Católica del Norte, Antofagasta, Chile.

Patricio Aroca. Instituto de Economía Aplicada Regional (IDEAR), Universidad Católica del Norte, Antofagasta, Chile.

RESUMEN | Diferentes trabajos señalan que, según la superficie de Chile y el nivel de desarrollo que ha alcanzado, la concentración de la actividad y población en la Región Metropolitana resulta excesiva. Además, durante las dos últimas décadas, la desigualdad espacial ha aumentado en el país, situación que podría afectar negativamente su crecimiento y que ha sido sistemáticamente ignorada en el diseño de las políticas públicas. Este trabajo muestra que diversos mecanismos de mercado, como la movilidad de los trabajadores y los flujos de comercio entre regiones, y muchas de las políticas públicas, tienden a favorecer las fuerzas concentradoras que han configurado la geografía económica de Chile durante las últimas décadas. Tales resultados hacen presente la necesidad de incorporar la "cuestión regional" de manera explícita y activa en la estrategia de desarrollo nacional, no solo como un problema de equidad, sino también como parte de las políticas para mejorar la eficiencia de la economía chilena.

PALABRAS CLAVE | concentración espacial, política regional, geografía económica.

ABSTRACT | Different studies show that, given the size of Chile and its level of development, there is an excess of concentration of population and activity in the Metropolitan Region. Furthermore, during the past two decades, spatial inequality has increased in Chile. This situation could negatively affect national growth, something that has been systematically ignored in the design of public policies. This article shows that some market mechanisms, such as labor mobility and inter-regional trade flows, and many public policies tend to strengthen the centripetal forces that have shaped the economic geography of Chile during the past decades. These results reveal the need to incorporate explicitly and actively the "regional problem" in the national development strategy, not only as an equity problem, but also as part of the policies oriented to improve the efficiency of Chilean economy.

KEY WORDS | spatial concentration, regional policy, economic geography.

Recibido el 9 de septiembre de 2010, aprobado el 31 de agosto de 2011.

E-mail: Miguel Atienza, miatien@ucn.cl | Patricio Aroca, paroca@ucn.cl

Se reconoce y agradece el apoyo del Fondo Nacional de Desarrollo Científico y Tecnológico (Fondecyt), proyecto 1090768: "Concentración territorial del desarrollo en Chile. Los efectos de la migración y la conmutación interregional”, y del Núcleo de la Iniciativa Científica Milenio "Ciencia Regional y Políticas Públicas". 


\section{Introducción}

La cuestión regional ha sido planteada en Chile desde los años sesenta, momento en que autores como Friedman y Stöhr (1966) afirmaban que "se estaba aproximando el momento en el que había que darle un gran apoyo a la investigación orientada a la economía regional", que permitiera el diseño de una política de planificación territorial fundamentada, acorde con las necesidades del país (p. 219). Dos décadas después, Larraín (1987) y Foxley (1987) señalaban la necesidad de pasar de la retórica a la acción en las políticas de descentralización. Tras más de veinte años desde esta afirmación, Chile es todavía uno de los países con mayor tasa de primacía del mundo. En 2010, más de 40\% de la población urbana del país reside en el área metropolitana de Santiago. Además, el grado de descentralización administrativa del país sigue siendo limitado. En este artículo se plantea que, en los más de cuarenta años que han pasado desde la propuesta de Friedman y Stöhr, la desigualdad regional ha pasado de ser un problema de equidad a otro donde, además, se ve comprometida la eficiencia del conjunto de Chile. Se muestra el riesgo que corre Chile de mantener los niveles de concentración existentes desde 1960 y cómo, pese a las declaraciones políticas a favor de las regiones, las fuerzas del mercado y las políticas públicas han tendido a mantener, si no a reforzar, la concentración espacial de la economía nacional en torno a la Región Metropolitana.

El artículo se organiza en cuatro partes. La primera analiza cómo, a medida que un país se desarrolla, la concentración espacial no solo plantea problemas de equidad, sino también de eficiencia. A continuación se describe la evolución de la concentración espacial en Chile durante las dos últimas décadas. En la tercera parte, se estudian los mecanismos que están contribuyendo a que Chile mantenga un patrón centro-periferia caracterizado por el exceso de concentración en la Región Metropolitana. En concreto, por un lado se analizan dos factores que, en teoría, deberían haber contribuido a reducir la concentración, como son la movilidad de los trabajadores y el comercio interno y externo; y por otro, se examinan algunas de las políticas aplicadas por los sucesivos gobiernos de Chile. Por último, las conclusiones plantean la necesidad de que la cuestión regional se incorpore de manera explícita y activa en la estrategia de desarrollo nacional como un medio para mejorar la eficiencia de la economía chilena.

\section{Concentración espacial, equidad y eficiencia}

Cada vez es más reconocida la fuerte relación existente entre el nivel de desarrollo de los países y su geografía económica (Banco Mundial, 2009). Es bien sabido que el proceso de desarrollo es espacialmente desequilibrado (Hirschman, 1958; Myrdal, 1957). Durante sus primeras etapas, la industrialización favorece el paso de una sociedad rural a una urbana y conduce a una geografía interna concentrada. Esta concentración espacial resulta eficiente para el conjunto de la economía, pues permite que las empresas localizadas en un mismo lugar se beneficien de rendimientos crecientes a escala, derivados de la proximidad. De estar dispersas, se verían imposibilitadas de ob- 
tener los mismos beneficios, principalmente en razón del reducido tamaño de la economía y de los altos costos del comercio, como efecto de la falta de infraestructura.

A medida que la economía se desarrolla, aparecen circunstancias favorables para la dispersión espacial. Así, las empresas localizadas en las regiones con mayor grado de concentración afrontan deseconomías externas puras de aglomeración y el alza relativa de la renta de la tierra y de los salarios. Al mismo tiempo, el crecimiento económico comporta un incremento de la demanda en los mercados locales y permite abaratar el costo del comercio interno, gracias a la inversión regional en infraestructuras. Además, el aumento de tamaño de la industria y sus actividades complementarias conducen a una mayor diversificación productiva y favorecen la aparición de economías a escala en las regiones periféricas. Con el desarrollo, por tanto, la desconcentración empieza a ser eficiente para la economía y es plausible esperar el comienzo de un proceso de dispersión regional de la industria. La llamada "hipótesis de Williamson" (1965) recoge estas ideas y propone que la relación entre la geografía económica (concentración) y el desarrollo económico se ajusta a una distribución cuadrática con forma de " $U$ " invertida. ${ }^{1}$

Los modelos de la Nueva Geografía Económica, donde el desarrollo se equipara a la disminución de los costos de comercio y la consiguiente integración del mercado interno (Fujita, Krugman \& Venables, 1999; Puga, 1999; Puga \& Venables, 1996; Van Marrewijk, 2005); han formalizado teóricamente la hipótesis de Williamson, la que también ha sido contrastada en estudios empíricos de corte transversal y de panel (Ades \& Glaeser, 1995; Brülhart \& Sbergami, 2009; Gaviria \& Stein, 2000; Henderson, 1999 \& 2003; Rosen \& Resnick, 1980; Wheaton \& Shishido, 1981). Son muy pocos, sin embargo, los estudios que abordan la experiencia de países concretos (entre ellos, Combes, Lafourcade, Thisse \& Toutain, 2008), y no está demostrado que esa regularidad empírica se cumpla de manera sistemática. Si bien se sabe que el proceso de concentración espacial que acompaña al desarrollo en sus etapas iniciales ocurre de manera muy rápida, la posterior dispersión de la actividad y de la población tiende a ser lenta y con distintos ritmos, dependiendo de las condiciones históricas y económicas de cada nación (Henderson, 1999).

La hipótesis de Williamson tiene implícita una relación estrecha entre desarrollo, medido como PIB per cápita, la concentración, la eficiencia y la equidad. Durante las primeras etapas del desarrollo, predomina un trade-off entre eficiencia y equidad espacial (Banco Mundial, 2009; Brülhart \& Sbergami, 2009), que puede llevar a que políticas orientadas a mejorar la distribución regional de los factores sean perjudiciales para el crecimiento. Gradualmente, a medida que un país se desarrolla, este efecto pierde fuerza, pudiendo llegarse a un escenario en el que una elevada concentración espacial no solo plantea problemas de equidad, sino también afecta negativamente el crecimiento (Davis \& Henderson, 2003). A este respecto, varios estudios (Brülhart \& Sbergami, 2009; Henderson, 1999 y 2003) han verifi-

1 Williamson (1965) se refiere, en realidad, a la convergencia en las rentas per cápita entre regiones, aunque su argumento también se ha empleado, especialmente por parte de la Nueva Geografía Económica, para la localización de las actividades. 
cado recientemente que el exceso de primacía de una o dos ciudades dentro del sistema urbano (medido en función del producto per cápita y el tamaño de los países) puede reducir significativamente la productividad y el crecimiento nacional.

Brülhart y Sbergami (2009) realizan una extensa investigación sobre los determinantes del crecimiento, eligiendo 18 entre 80 variables que resultaron robustas en una cantidad importante de trabajos que intentaban explicar el crecimiento regional en distintos países. A este conjunto agregaron la concentración de la población medida a través de un índice de primacía (Urban750), calculado como la proporción de la población que vive en ciudades de tamaño superior a 750.000 habitantes, y utilizado como una medida aproximada del grado de concentración espacial de la economía. Uno de los principales resultados de este estudio se muestra en la Figura 1, donde se aprecia que el signo de la relación entre concentración y crecimiento depende del nivel de producto per cápita del país. La concentración afecta positivamente el crecimiento para niveles bajos de producto per cápita, mientras que, pasado cierto nivel, esta relación cambia de signo o no es significativa. Más específicamente, los autores utilizan una base de datos de 105 países y muestran que el umbral del producto per cápita donde esta relación cambia de signo es de US $\$ 10.048$, estimados en paridad de poder de compra de $2006 .^{2}$ Es decir, en países con un producto per cápita superior a US\$10.000, sería recomendable reducir la concentración para lograr mayor crecimiento.

FIGURA 1 | Efecto de la concentración urbana sobre el crecimiento

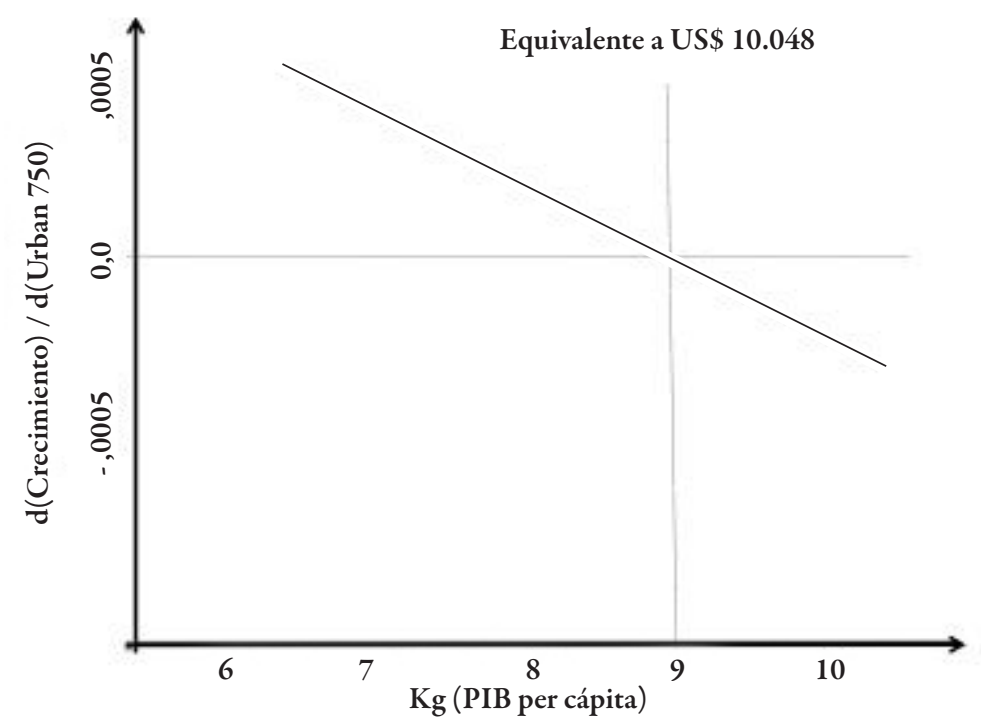

Fuente Autores a partir de Brülhart y Sbergami (2009).

Trabajos anteriores, como el de Wheaton y Shishido (1981), identificaron un umbral de producto per cápita ligeramente inferior, de US\$8.384. 
Desde un punto de vista estático, la pérdida de eficiencia provocada por el exceso de concentración tiene su origen, por un lado, en la mala asignación de recursos hacia grandes aglomeraciones expuestas a deseconomías a escala, congestión y altos costos de inversión per cápita en infraestructura; y, por otro, en el desaprovechamiento de las economías a escala y la deficiente formación de capital en las áreas periféricas. Desde una perspectiva dinámica, estas pérdidas provienen del hecho de que las grandes aglomeraciones desvían recursos que podrían dedicarse a la inversión e innovación en actividades productivas y los emplean para intentar mantener la calidad de vida en entornos locales congestionados (Duranton \& Puga, 2001; Henderson, 2003).

Como ya señaló Hirschman (1958), las políticas públicas desempeñan un papel determinante en el paso de una geografía económica polarizada a otra más equilibrada. Se ha verificado empíricamente que el marco institucional y determinadas políticas pueden favorecer el exceso de concentración, generando el fenómeno conocido como "favoritismo", esto es, la existencia de incentivos económicos y políticos para que los recursos se dirijan mayoritariamente a las grandes aglomeraciones (Ades \& Glaeser, 1995; Davis \& Henderson, 2003). Por el contrario, otras políticas, como la inversión en infraestructura regional, una mayor descentralización fiscal y mayores niveles de democracia facilitan la desconcentración de la actividad desde las regiones centrales a las periféricas (Davis \& Henderson, 2003). Acciones de este tipo contribuyen a una mayor equidad espacial, pero también pueden tener una incidencia positiva en el crecimiento de países de rentas medias.

\section{Concentración espacial, equidad y eficiencia en Chile}

Según el Censo de Población y Vivienda de Chile de 2002, la Región Metropolitana concentra el $43 \%$ de la población urbana del país y cerca del $60 \%$ de la mano de obra ocupada en zonas urbanas. A este respecto, diversos estudios (Henderson, 1999 y 2003; Brülhart \& Sbergami, 2009) señalan que, si se tiene en cuenta el producto per cápita y el tamaño del país, medido según su población urbana, Chile se encuentra en el grupo de países que presentan un exceso de concentración, junto a otros como Argentina, Perú, México, Argelia y Tailandia (Henderson, 2003). Chile habría superado en torno al año 2002 el umbral de renta per cápita estimado por Brülhart y Sbergami (2009), a partir del cual el exceso de concentración afecta negativamente el crecimiento (Figura 2). ${ }^{3}$ Por tanto, cabe pensar que la actual concentración espacial en la Región Metropolitana puede estar afectando negativamente la productividad y capacidad de crecimiento del país. Es relevante, por tanto, preguntarse si Chile ha empezado a desconcentrarse espacialmente según lo planteado por la hipótesis de Williamson o si, por el contrario, todavía prevalecen las fuerzas centrípetas y las políticas que favorecen la persistencia de un patrón centro-periferia que, en estos momentos, puede resultar ineficiente.

3 La Figura 2 incluye también el año en que Chile alcanzó el umbral de producto per cápita estimado por Wheaton y Shishido (1981). Según dicha estimación, en torno a 1986 el país tenía un producto per cápita para el cual la concentración ya podría estar afectando negativamente al crecimiento. 
FIGURA 2 | Evolución del PIB per cápita de Chile 1990-2008 (en PPP de 2006)

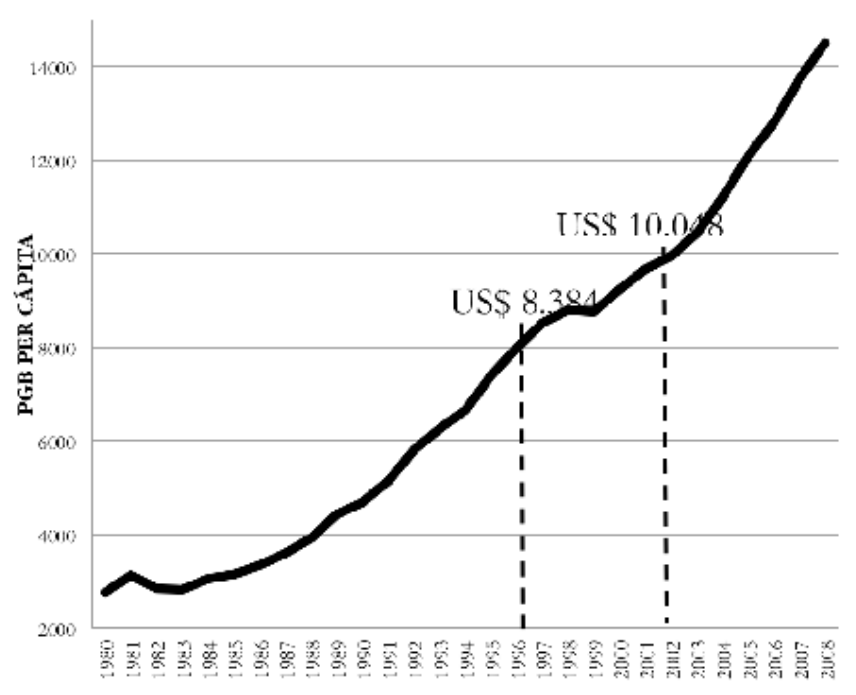

FUENTE ELABORACIÓN PROPIA.

Nota PPP = Purchasing Power Parity: PARIdad de POder adquisitivo.

Para tratar de responder la pregunta recién planteada, es conveniente una aproximación inicial de carácter histórico que permita evaluar de qué manera ha evolucionado la geografía económica chilena a lo largo de su desarrollo económico. Como señalan Geisse y Valdiva (1978), la urbanización chilena fue temprana y espacialmente desconcentrada, por no estar basada en el crecimiento de la industria, sino más bien en la explotación de actividades primarias (agrarias y mineras) orientadas a la exportación. En 1907, el 38\% de la población chilena era urbana y la ciudad de Santiago representaba el $27 \%$ de esa población y algo más del $10 \%$ de la población total, una tasa de primacía llamativamente baja. El proceso de industrialización, que dio lugar a una sociedad urbanizada y al patrón espacial centro-periferia que hoy conocemos, alcanzó todo su potencial al alero de la política de industrialización por sustitución de importaciones que caracterizó la economía chilena a partir de los años treinta y hasta comienzos de los años setenta del siglo XX. En 1970, el Gran Santiago concentraba aproximadamente el 35\% de la población total y el $44 \%$ de la población urbana (Geisse \& Valdivia, 1978; Miranda Muñoz, 1997). Ya en ese año, Henderson (2003) estimaba que el grado de primacía del Gran Santiago era excesivo en función del PIB per cápita alcanzado por el país y el tamaño de su población urbana.

El crecimiento posterior y los cambios políticos y estructurales acaecidos en la sociedad y la economía chilenas hacían previsible un proceso de progresiva desconcentración de la actividad económica. Entre los factores que, desde un punto de vista teórico, podrían haber contribuido a una distribución espacial más equitativa cabe destacar la creciente apertura comercial (Ades \& Glaeser, 1995; Krugman \& Livas, 1996); el progresivo aumento del producto per cápita; la reducción de los 
costos de transporte internos; la creciente terciarización; y, desde el comienzo de los años noventa, el paso de un régimen dictatorial a uno democrático, que declaró el objetivo político de promover una mayor descentralización administrativa. Sin embargo, lejos de producirse una reducción de la primacía de la Región Metropolitana, durante las dos últimas décadas parece haberse consolidado el patrón espacial polarizado que surgió del proceso de industrialización.

A modo de ilustración de lo ocurrido durante las dos últimas décadas y siguiendo el trabajo de Combes et al. (2008) para Francia, se presenta la evolución del índice de Theil espacial en Chile. Este índice permite calcular la desigualdad espacial entre regiones tomando como punto de referencia una distribución uniforme de la actividad económica, según la siguiente expresión:

$$
T=\sum_{r=1}^{R} \frac{A_{r}}{A} \log \frac{A_{r}}{A / R} \quad \text { siendo } \quad A=\sum_{r=1}^{R} A_{r}
$$

donde $A_{r}$ representa el nivel de actividad en la región $r$ y $A$ representa el total de actividad nacional. El índice oscila entre un valor mínimo 0 , cuando la actividad se distribuye de forma uniforme entre regiones, y un valor máximo, en el caso de que toda la actividad se concentrase en una sola región, equivalente al $\log R=1,114$ (siendo $R=13$ regiones).

La Figura 3 ilustra la evolución del índice de Theil de tres variables representativas de la actividad económica entre 1986 y 2006: el producto, el empleo y la población. En líneas generales, puede observarse que la desigualdad espacial ha tendido a aumentar ligeramente durante el periodo analizado, con algunas diferencias según la variable considerada. El mayor grado de desigualdad entre regiones se observa en el producto, aunque es esta la única variable que ha experimentado una tendencia decreciente a partir del año 1993. Por su parte, la desigualdad en el empleo aumentó durante la segunda mitad de los ochenta y ha mantenido, desde entonces, un nivel relativamente estable. Por último, la desigualdad en población ha sido la que ha presentado menos variaciones, mostrando una leve tendencia al alza.

Los resultados recién presentados hacen pensar que en Chile no se cumple, por el momento, la hipótesis de Williamson y que algunos factores contribuyen al sostenimiento de una geografía económica excesivamente concentrada, lo que puede estar limitando la eficiencia de la economía nacional, una relación hasta ahora ignorada por las políticas públicas. En este sentido, los resultados obtenidos a partir de las datos proveídos por Brülhart y Sbergami (2009) muestran que en países sudamericanos como Perú, Argentina, Chile, Uruguay y Venezuela el efecto de la concentración - medido a través de un índice de primacía de la población urbana- sobre la tasa de crecimiento de largo plazo es significativo y negativo (Figura 4), mientras que lo mismo no ocurre para el resto de los países sudamericanos. ${ }^{4}$

4 La línea vertical de la Figura 4 señala el umbral del PIB per cápita a partir del cual el impacto negativo de la primacía en la tasa de crecimiento es estadísticamente significativo, teniendo en cuenta los márgenes de error de la estimación. 
Adicionalmente, estos resultados son consistentes con otros presentados en la literatura en periodos previos, donde Chile aparece sistemáticamente como uno de los países más concentrados del mundo (Henderson, 2003).

\section{FIGURA 3 | Índice de Theil de empleo, producto y población, 1986-2006}

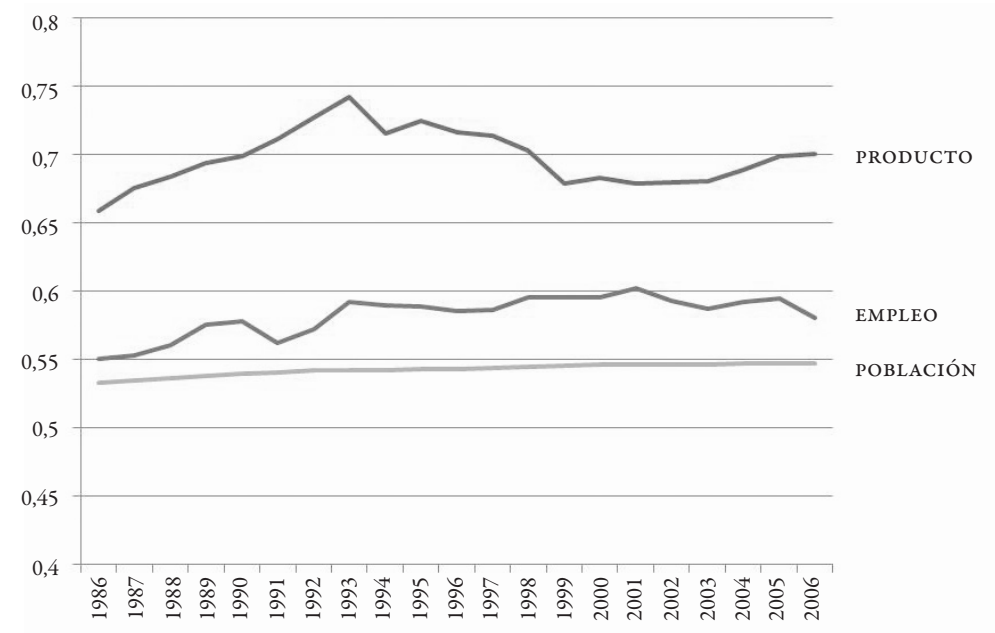

FUENTE ELABORACIÓN PROPIA.

FIGURA 4 | Relación entre crecimiento y concentración en países sudamericanos



FUENTE Elaboración PROPIA A PARTIR DE DATOS Y Códigos PROPORCIONADOS POR BRÜLHART Y SBERGAMI (2009). 


\section{Mecanismos concentradores de la población y la actividad económica en Chile ${ }^{5}$}

Armstrong y Taylor (2003) y Duranton (2009) sostienen que en los países de desarrollo medio donde existe una ciudad o región dominante, como la Región Metropolitana en Chile, los mercados y las instituciones ponen en marcha un conjunto de mecanismos concentradores. En dichas regiones existe una congestión creciente de la infraestructura y los servicios públicos, y — según muestra la experiencia - el mercado, en conjunto con el sector público, tiende a resolver este problema mediante una mayor inversión y provisión per cápita de estos bienes, en lugar de reducir la demanda de ellos mediante un desarrollo territorial más equilibrado (Richardson, 1987). A continuación se describen algunos de los mecanismos de mercado y de las políticas que en Chile están contribuyendo a promover la desigualdad territorial y la concentración en torno a la Región Metropolitana y que podrían estar afectando la eficiencia del país.

\section{Comercio interregional e internacional}

Varios autores han señalado que las políticas de apertura comercial favorecen la desconcentración de la actividad productiva (Livas \& Krugman, 1992; Hanson, 1994), debido a que las empresas que se orientan a la exportación y que dependen de las importaciones para la compra de insumos no tendrán incentivos para localizarse en el centro nacional, donde sus encadenamientos son débiles y afrontan deseconomías de aglomeración. La apertura comercial llevaría, por tanto, a un proceso de desconcentración que reduce la primacía y genera sistemas urbanos más equilibrados.

La hipótesis de debilitamiento de los encadenamientos entre la periferia y el centro en los países exportadores es puesta en duda por trabajos como el de Hulu y Hewings (1993) para el caso de Indonesia. Estos autores plantean una metodología para estimar cómo se distribuye el impacto de los proyectos o políticas que implican gasto público entre los cinco grandes conjuntos de islas que componen Indonesia (Sumatra, Java, Kalimantan, Sulawesi y las Islas del Este), teniendo en cuenta las relaciones comerciales entre ellos. Debido a estas relaciones, el impacto de un proyecto desarrollado en uno de los cinco conjuntos de islas se difunde también hacia el resto. Esta difusión no es homogénea en el espacio y depende del patrón de comercio entre las regiones. Las regiones que exportaban más al resto del país recibían un mayor impacto de proyectos realizados en otras regiones, mientras que las regiones importadoras retenían un menor impacto de los proyectos realizados en su territorio. Los resultados son sorprendentes. Por ejemplo, el 94,2\% del impacto total de un proyecto en el sector bancario y financiero del principal centro nacional, Java, lograba quedarse en esa región, mientras que las Islas del Este del archipiélago capturaban menos del 10\% del impacto total de un proyecto del sector electricidad, gas y agua realizado en su territorio.

5 Esta sección se basa parcialmente en Aroca (2009). 
Haddad (1999) y Hewings (2009) desarrollan y aplican un modelo de equilibrio general computable que permite evaluar los resultados regionales del Mercosur en Brasil. Aunque, en general, los datos muestran que Brasil es probablemente el país más beneficiado con el acuerdo, la distribución de estos beneficios en el interior del país es muy desigual. Todos los sectores económicos del centro-sur del país crecen gracias al acuerdo, mientras que en el nordeste brasileño, la zona menos desarrollada del país, la mayoría de los sectores se ven afectados negativamente. Tanto en Indonesia como en Brasil, la explicación de estos resultados se basa en los patrones de comercio existentes dentro del país. Java en Indonesia y São Paulo en Brasil presentan grandes superávit comerciales con el resto de las regiones del país, y no así con el resto del mundo.

Un patrón similar se observa en el caso de Chile. La Figura 5 muestra las únicas cifras estimadas de comercio interregional disponibles en Chile, que corresponden al año 1996, y las de las regiones con el resto del mundo, para el mismo año. Por un lado, se aprecia que la Región Metropolitana presenta un gran superávit comercial respecto del resto de las regiones del país, mientras que tiene un déficit proporcionalmente mayor con el resto del mundo. Es decir, Santiago importa bienes del resto del mundo y exporta hacia las regiones. Por otra parte, las regiones se encuentran en un escenario opuesto: tienen significativos superávit comerciales con el resto del mundo, mientras que presentan déficit con el resto del país. Por lo anterior, como en Indonesia y Brasil, es razonable pensar que, pese al alto grado de apertura comercial del país, cuando se hace un proyecto en una región de la periferia, una proporción importante de los gastos de ese proyecto terminará en la Región Metropolitana. Por el contrario, un proyecto realizado en la Región Metropolitana importará muy poco del resto de las regiones, y probablemente tendrá un impacto más significativo en las importaciones del resto del mundo. En definitiva, el patrón de comercio interno en Chile no manifiesta un debilitamiento de los encadenamientos productivos de la periferia con el centro, como plantean algunos autores. La apertura comercial no parece haber actuado en el país como una fuerza centrífuga, sino más bien al contrario, debido a que la concentración de la demanda de bienes y servicios importados en la Región Metropolitana hace que proyectos que se realizan en todo el país incrementen significativamente la demanda de la Región Metropolitana, pero no del resto de las regiones. Del mismo modo, la distribución de los beneficios del comercio internacional terminará concentrándose en torno al ya concentrado centro del país.

Además del mantenimiento de la fuerte primacía de la Región Metropolitana, una implicación relevante para las regiones periféricas de este patrón de comercio interno es su falta de diversificación productiva. En su mayoría estas regiones continúan estando fuertemente especializadas, lo que pone en riesgo su sustentabilidad económica y social, especialmente en aquellas zonas intensivas en la explotación de recursos naturales que dependen fuertemente de los ciclos de la demanda internacional. 
FIGURA 5 | Comercio regional e interregional en Chile, 1996 (millones de pesos)

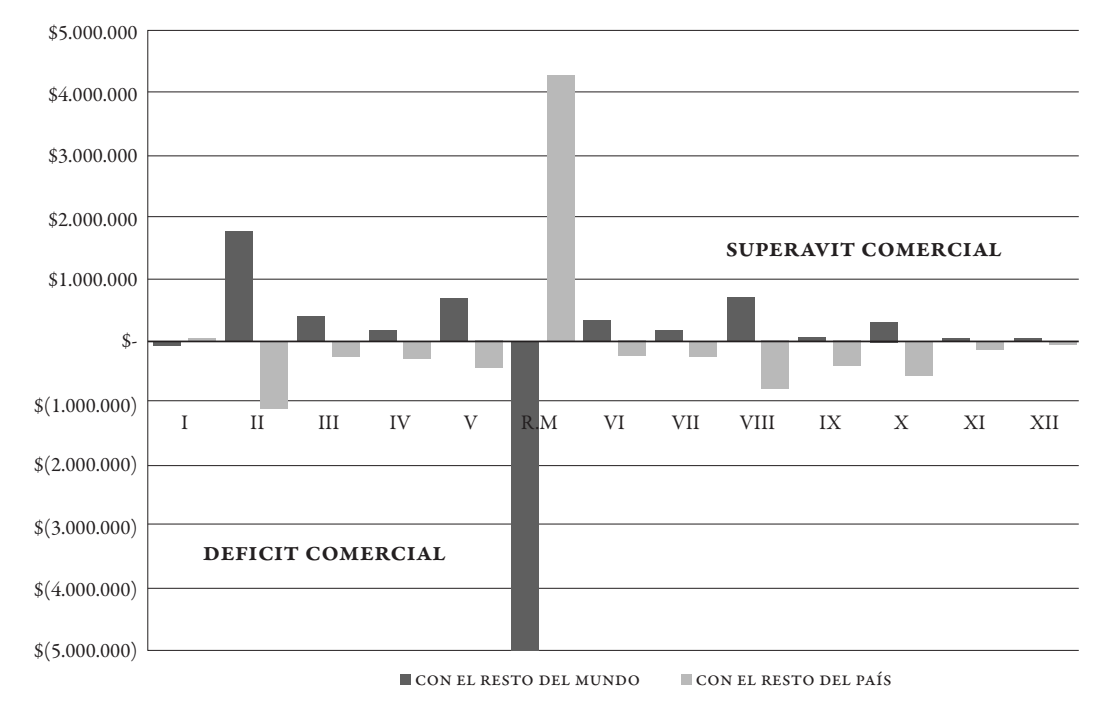

FUENTE ELABORACIÓN PROPIA CON BASE EN LAS MATRICES REGIONALES DE INSUMO-PRODUCTO 1996, MINISTERIO de Planificación y Cooperación (Mideplan), Chile.

\section{Movilidad laboral: migración y conmutación interregional}

La teoría económica tradicional plantea que, en una economía de mercado, la migración laboral actúa como un mecanismo que reduce las desigualdades regionales en salarios y desempleo. Se supone que los trabajadores dejarán las regiones con alto desempleo e irán a aquellas donde tienen mayores posibilidades de encontrar trabajo. De este modo, se reduce el desempleo en la región de origen, aumenta en la de destino y, al mismo tiempo, se igualan los salarios que se pagan en la economía, dando como resultado menores desigualdades territoriales. Los supuestos de la teoría clásica de la migración entre regiones quedan en entredicho al observar la dirección que siguen los flujos de trabajadores y nuevos modos de movilidad, que pueden llegar a tener más relevancia que la migración.

En Chile existe una forma de movilidad laboral que en el Censo de Población y Vivienda de 2002 resultó ser casi el doble que la migración y que debilita la capacidad de contribuir a la convergencia que se le atribuye a la movilidad del trabajo. Aroca y Atienza (2008) la denominan "conmutación interregional o de larga distancia”, y consiste en que una proporción importante de trabajadores opta por separar la región de trabajo de la región de residencia. Un ejemplo extremo de este fenómeno es la Región de Antofagasta, que, según el Censo de 2002, recibía16.500 trabajadores desde otras regiones, lo que representa más de $10 \%$ de la fuerza laboral local. Esta situación es promovida por el código laboral, al permitir establecer sistemas de turnos en todas las 
actividades. Estos turnos van desde trabajar doce horas durante cuatro días seguidos y descansar tres, hasta trabajar veintidós días consecutivos y descansar ocho.

Los conmutantes laborales, además, tienen en promedio ingresos más altos que los trabajadores locales, por lo que el monto de salarios que se llevan hacia sus regiones de origen puede ser muy significativo. Aroca y Atienza (2008) estiman que el monto de ingresos que se llevan los conmutantes desde la Región de Antofagasta podría ser superior en más de cuatro veces al monto que esa región percibe por concepto de Fondo Nacional de Desarrollo Regional. También muestran que solo las regiones extremas de Chile tienen conmutación neta positiva, mientras que las centrales tienen conmutación neta negativa. Es decir, la proporción de trabajadores que van desde el centro del país a trabajar a las regiones extremas (Tarapacá y Antofagasta en el norte y Aysén y Magallanes en el sur) es superior a la de quienes van desde las zonas extremas a las regiones del centro (Cuadro 1). Esta movilidad laboral es, por tanto, altamente concentradora de ingresos en torno a las regiones centrales del país y actúa como una fuente de desigualdad territorial. A ello deben añadirse los diversos problemas territoriales que provoca esta forma de movilidad, como, por ejemplo, la desestructuración de la vida familiar en las regiones de origen, la falta de identidad y el impacto negativo en la vida comunitaria en las regiones de destino, y los problemas de salud que provoca en los trabajadores el sistema de turnos (De Laire, 1999; Storey, 2010).

CUADRO 1 | Tasas netas de recepción regional de conmutantes, 2002 (porcentajes)

\begin{tabular}{l|c|c|c}
\hline REGIÓN & CONMUTAN FUERA & CONMUTAN DENTRO & $\begin{array}{c}\text { TASA NETA DE } \\
\text { RECEPCIÓN DE } \\
\text { CONMUTANTES }\end{array}$ \\
\hline I & 2,20 & 3,70 & 1,50 \\
II & 1,40 & 11,00 & 9,60 \\
III & 5,30 & 6,10 & 0,80 \\
IV & 5,00 & 2,50 & $-2,40$ \\
V, VI Y RM & 1,00 & 0,70 & $-0,30$ \\
VII & 3,00 & 2,40 & $-0,60$ \\
VIII & 3,20 & 1,90 & $-1,40$ \\
IX & 3,80 & 2,10 & $-1,70$ \\
X & 1,30 & 2,40 & 1,10 \\
XI & 0,60 & 6,20 & 5,60 \\
XII & 0,80 & 3,70 & 2,90 \\
\hline
\end{tabular}

FUENTE ELABORACIÓN PROPIA A PARTIR DEL CENSO 2002.

Nota: Las regiones V, de Valparaíso, y VI, del Libertador General Bernardo O'Higgins, Se unen a la Región METROPOLITANA POR CONSIDERAR QUE LA CONMUTACIÓN ENTRE ELLAS TIENE UN CARÁCTER FUNDAMENTALMENTE METROPOLITANO.

\section{Políticas concentradoras de población y actividad económica del Estado}

Junto a los mecanismos de mercado mencionados existe una extensa evidencia de que las políticas promovidas por el Gobierno de Chile han contribuido a la excesiva concentración existente en el centro del país. Geisse y Valdivia (1978) y Carlin (2009) señalan que la política de comercio exterior proteccionista aplicada desde 
la década de los treinta a la de los setenta, resultó fuertemente concentradora de la población en torno a Santiago. Esta situación, como se ha mostrado, no se ha revertido con la apertura comercial posterior. A ello han contribuido, junto con el patrón de comercio entre regiones, una variedad de políticas concentradoras de la población y la actividad económica propiciadas por los diferentes gobiernos del país, tal y como señala Benavente (2007). A continuación se analizan brevemente algunas de las políticas de entes del Estado que han tenido efectos concentradores y que muestran la disonancia existente entre lo declarado y lo realizado en torno al problema de la concentración y las desigualdades regionales, así como la indiferencia de los responsables de las políticas relacionadas con respecto a este problema.

\section{El Fondo Nacional de Desarrollo Regional (FNDR)}

La ley que creó el Fondo Nacional de Desarrollo Regional (FNDR) establece que "el Fondo Nacional de Desarrollo Regional es un programa de inversiones públicas, con finalidades de compensación territorial, destinado al financiamiento de acciones en los distintos ámbitos de infraestructura social y económica de la región, con el objeto de obtener un desarrollo territorial armónico y equitativo", a cargo de la Subsecretaría de Desarrollo Regional (Subdere), dependiente del Ministerio del Interior.

Después de una década en que claramente las desigualdades regionales en el ingreso de las personas no han disminuido, llama la atención que el mayor aumento en la distribución del FNDR realizado por la Subdere entre 2003 y 2006 lo haya experimentado la Región Metropolitana, con un 160\%. En contraste, el incremento para el resto de las regiones promedia un $44 \%$. Esto significa que la región con mayores ingresos de la economía ha aumentado su participación más del ciento por ciento por encima del resto de las regiones en el fondo creado con el objetivo de reducir las desigualdades regionales. En contraste, otras regiones que requieren de mayor gasto público que promueva una mayor equidad territorial, reciben un porcentaje considerablemente menor. Al respecto, es importante aclarar que, aunque el FNDR no es el fondo público más importante que distribuye el Estado, es el único creado con el objetivo de reducir las diferencias regionales, por lo que no hay razón para esperar que los otros fondos estatales tengan un tratamiento diferente cuando su objetivo principal no es el de promover equidad territorial.

La Organización para la Cooperación y el Desarrollo Económicos (OCDE, 2009) señala que existen dos tipos de FNDR, uno que es de libre disposición de las regiones, el cual depende de la selección de proyectos que realizan los gobiernos regionales; y otro, llamado Provisiones, que les da a los gobiernos regionales menor control sobre los proyectos y el territorio donde realizarlos, ya que no siguen el reglamento de distribución del FNDR original y se encuentran fuertemente influenciados por las decisiones del gobierno central. De hecho, son estas Provisiones las que explican principalmente el crecimiento de las asignaciones a la Región Metropolitana, contrastando con el objetivo de compensación territorial del FNDR original. En este sentido, las Provisiones podrían interpretarse como una manera de ignorar el reglamento, ya que su distribución sigue un patrón que se define en el momento de crearlas. La Universidad de Los Lagos (2010) muestra que, desde 
1997, las Provisiones han seguido una tendencia creciente, en desmedro del FNDR de libre disposición, llegando a representar en 2009, un 52\% del total del FNDR asignado a las regiones. Ese año existían dieciséis Provisiones diferentes, lo que podría explicar el significativo incremento de la proporción que va a la Región Metropolitana en perjuicio de las demás regiones del país.

\section{La centralización del gasto público}

Waissbluth (2006) desarrolla un análisis de la evolución de la inversión pública que, en la Figura 6, se complementa con información de Mideplan. Los montos de inversión pública comunal y regional se deciden en la comuna y en la región, mientras que los montos sectoriales lo hacen en los ministerios del país. La tendencia presenta un crecimiento sostenido de la inversión pública comunal y regional hasta el año 2000, que alcanza el 41,2\%, muy superior al 26\% de 1992. Sin embargo, a partir del año 2000, el porcentaje de decisión local y regional comienza a caer, alcanzando en 2007 solo un 29,8\% del total de la inversión. Es decir, se retrocede a niveles levemente superiores a los de 1992. Estos antecedentes contradicen la idea de la búsqueda de mejorar la eficiencia en la asignación de recursos a través de dar poder de decisión a las regiones y comunas respecto de aquellos proyectos en que invertir es más rentable socialmente; en particular, los orientados a la provisión de infraestructuras y bienes públicos que mejoren la calidad de vida y hagan más atractivas esas regiones y comunas. Más bien, como el poder central está en Santiago, cabe pensar que la asignación para la Región Metropolitana será más eficiente, pues quienes deciden el destino del gasto público conocen con mayor precisión los problemas de la ciudad que habitan.

\section{FIGURA 6 | Inversión pública efectiva comunal, regional y sectorial}

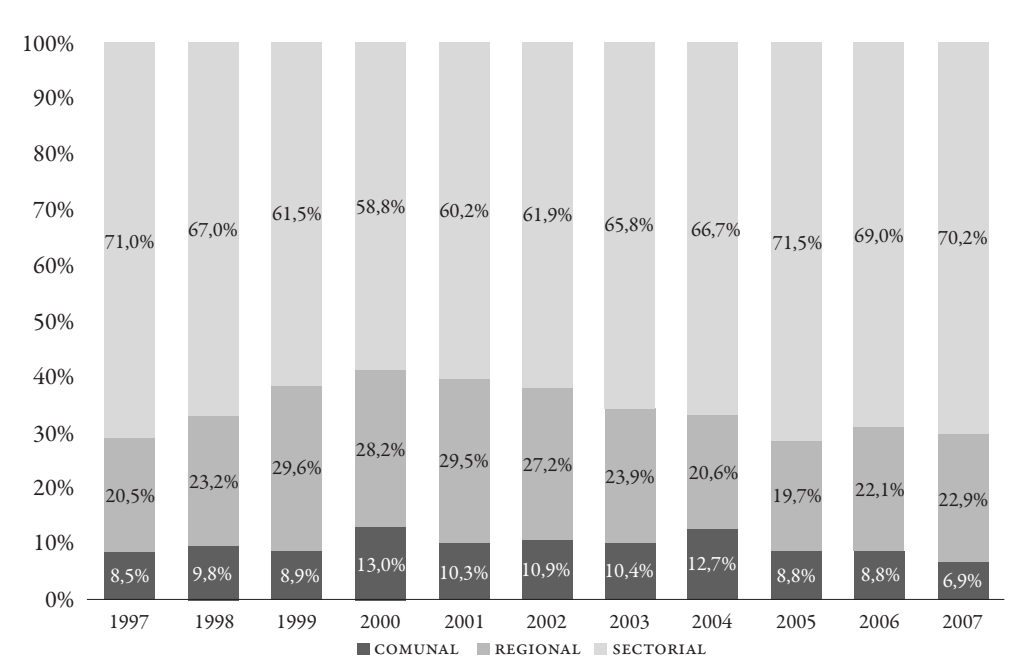

FUENTE ELABORACIÓN PROPIA CON DATOS DE MIDEPLAN. 


\section{Capital humano avanzado y las becas}

Una de las grandes diferencias entre la Región Metropolitana y el resto de regiones de Chile tiene que ver con el nivel de capacitación de sus trabajadores y las funciones que estos cumplen, aspectos que, a su vez, se manifiestan en mayores salarios y un mayor atractivo de la Región Metropolitana para el conjunto de la población. Es conocida la concentración histórica de las principales universidades del país en el área metropolitana de Santiago y el atractivo que ellas ejercen sobre buena parte de los estudiantes, que finalmente deciden residir en esta zona. Tal situación, unida al hecho de que la Región Metropolitana es el principal y casi único nodo de vinculación del país con la economía global, además de ser la sede de las principales empresas del país, ha dado lugar a un patrón de división espacial del trabajo que se caracteriza por la fuerte especialización de la Región Metropolitana en las ocupaciones de mayor contenido cognitivo simbólico, mientras que el resto de las regiones tiende a estar especializado en funciones de tipo rutinario, físico y que requieren una calificación media o baja (Atienza, Lufín \& Sarrias, 2010; Lufín \& Atienza, 2010). Al mismo tiempo, Paredes (2011) estima que los diferenciales comunales de salario se explican fundamentalmente por la calificación de los trabajadores, lo que refuerza el atractivo de la Región Metropolitana.

Estos resultados muestran la necesidad de las regiones periféricas del país de atraer un mayor número de trabajadores calificados que contribuyan al desarrollo de las ciudades intermedias del sistema urbano chileno, incrementando su capacidad de innovación y potenciando su productividad, así como el desarrollo de una demanda residencial más diversificada. Si bien el gobierno ha tomado conciencia de la relevancia de fomentar la formación de capital humano avanzado, la aplicación de las políticas con este fin ha carecido, hasta ahora, de un enfoque que considere la situación de las regiones periféricas.

El gobierno ha creado la Beca Presidente de la República para financiar estudios de posgrado, especialmente doctorados, en el extranjero. Recientemente su nombre ha cambiado a Beca Chile y ha tenido un incremento significativo en los montos asignados, especialmente después de que el Consejo Nacional de Innovación estableciera como uno de los cuellos de botella del proceso de desarrollo chileno, la escasez de capital humano avanzado. Desde 1981 hasta 2006, se han asignado 2.274 becas y se espera asignar 25.000 en los próximos veinticinco años. La ministra de Mideplan decía en una entrevista sobre las becas que "hasta el año 2006, desde que existen las becas, el $73 \%$ de los becarios fueron varones y el $27 \%$ mujeres. El año 2006, cuando seleccionamos con currículum ciego, la cifra cambió a 53\% de varones y $47 \%$ de mujeres". Sin embargo, no hay ninguna mención en su análisis que explique por qué se ha concentrado la asignación en la Región Metropolitana, donde vive alrededor del $40 \%$ de la población del país y que en 2006, en la primera lista dada a conocer de los seleccionados, se adjudicó el 76\% de las becas. En 2007 esta situación mejoró, si bien el porcentaje asignado a la Región Metropolitana alcanzó el $62 \%$. 
Otro problema que acentúa la desigualdad regional tiene relación con el hecho de que para los nuevos doctores o posgraduados, es más atractivo incorporarse a equipos de Santiago que de regiones, ya que tienen mayor experiencia y obtienen mayores fondos para realizar investigación. Siendo así, una proporción importante de aquellos que obtienen la beca Presidente de la República provenientes de regiones se queden trabajando en Santiago, en desmedro de las regiones periféricas, que padecen una fuga de cerebros. Recientemente se ha propuesto que los becarios devuelvan la beca trabajando en regiones por un periodo de tiempo similar al que estuvieron estudiando. Esta propuesta no ha prosperado y, por lo tanto, es de esperar que el capital humano avanzado se siga concentrando en la Región Metropolitana, como lo ha hecho en el pasado.

\section{La política monetaria del Banco Central}

El Banco Central de Chile es una institución estatal, independiente del gobierno, encargada de mantener un nivel bajo de inflación. El 3\% de inflación anual es el objetivo fijado de la política monetaria. El principal instrumento utilizado ha sido la tasa de interés, la cual se aumenta cuando la inflación está por encima del objetivo, con el fin de incrementar el costo del endeudamiento y reducir, a través de este hecho, el consumo de las familias, con la consiguiente presión para que los precios bajen. Esta política nacional no es neutra espacialmente y tiene distintos efectos en cada una de las regiones (Bravo, García, Mies \& Tapia, 2003), más aún si se tiene en cuenta que en Chile la medición de la inflación se realiza solamente con datos del Gran Santiago. Es decir, tanto la canasta como los precios utilizados para medir la inflación se basan exclusivamente en información de familias y negocios ubicados en la Región Metropolitana. ${ }^{6}$

Dada la heterogeneidad territorial de la economía chilena, caracterizada por una fuerte especialización productiva del norte en minería, del centro en manufacturas y servicios, y del sur en agricultura y pesca, es muy probable que los shocks provocados por cambios en precios en Santiago también se produzcan en el resto del territorio, aunque con diferencias en cuanto a su magnitud y dirección. Esto es, la tasa de interés que actúa como un freno o acelerador eficiente para Santiago, no necesariamente resulta adecuada para todo el territorio. Puede haber regiones que reciban un freno antes de necesitarlo o un acelerador tardíamente, porque su inflación no sigue la dinámica de la Región Metropolitana. La puesta en marcha del nuevo Índice de Precios al Consumidor podría ayudar a reducir, al menos parcialmente, los efectos negativos que tiene para las regiones el no considerarlas en la construcción de la canasta y la elección de los lugares de compra. Sin embargo, aún es necesaria una política fiscal complementaria emanada desde el gobierno, que reduzca las ineficiencias que se producen en aquellas regiones que tienen presiones inflacionarias por debajo del promedio del país, y contenga los incrementos de costo de vida en aquellas otras donde dichas presiones están por sobre ese promedio.

6 El Instituto Nacional de Estadísticas (INE) ha cambiado esta modalidad, al considerar familiares de regiones en la nueva encuesta de presupuesto familiar a partir de 2009, y también recolección de precios regionales; sin embargo, el diseño se ha realizado de manera tal que no permite el cálculo de inflación regional (INE, 2009). 
Sarrias y Aroca (2011) complementan este resultado mostrando que los ciclos económicos de las regiones están menos sincronizados a medida que más se alejan de la Región Metropolitana. Este resultado es otra señal clara de la necesidad de realizar política regional destinada a mejorar la eficiencia en las regiones, ya que actualmente el diseño de política monetaria y fiscal apunta principalmente a hacer eficiente el desempeño de la Región Metropolitana y de aquellas otras regiones que más se le parecen.

\section{La estrategia nacional para la competitividad}

Esta estrategia, propuesta por el Consejo Nacional de Innovación para la Competitividad en 2007, es un claro ejemplo de la diferencia entre la retórica pública, favorable al desarrollo regional, y la acción, que mantiene activas las fuerzas concentradoras. En principio, se podría pensar que dicha estrategia tiene una orientación que favorece el desarrollo regional, ya que uno de sus ejes principales es la promoción de potenciales clusters regionales identificados en los sectores de externalización de servicios: piscicultura, turismo, minería, porcicultura y avicultura, alimentos procesados para el consumo humano, industria frutícola primaria y servicios financieros.

La estrategia nacional para la competitividad apunta a aprovechar las ventajas de estos incipientes clusters, basados en su mayoría en recursos naturales, para generar en torno a ellos prácticas de innovación, aumentar la productividad y transformar las ventajas estáticas en dinámicas, siendo estas últimas resultado de la construcción y dominio privilegiado de conocimientos y técnicas que se profundizan con el tiempo. Se pretende también desarrollar nuevas actividades, vinculando la base exportadora de Chile a productos con mayor valor y al desarrollo de servicios y bienes especializados, orientados a la satisfacción de las necesidades de los clusters más intensivos en recursos naturales, impulsando así una mayor diversificación de la estructura productiva.

El estudio territorial para Chile de la OCDE (2009) ha planteado serias dudas sobre las ventajas que la estrategia recién descrita puede tener para las regiones chilenas no metropolitanas. Dicho estudio señala, por un lado, que la mayoría de los clusters elegidos, especialmente los situados en las regiones periféricas, se basan en sectores ya maduros e ignoran las potencialidades de varias regiones del país. Por otro lado, se trata de actividades que no requieren de un capital humano de alta calificación, por lo que los incentivos locales para mejorar la composición de sus mercados laborales son reducidos. Debe añadirse, además, que la concentración relativa de las funciones gerenciales y profesionales de las actividades seleccionadas en el área metropolitana de Santiago puede fortalecer principalmente el centro económico del país, perdiendo los clusters su carácter territorial (Lufín \& Atienza, 2009; Atienza, Lufín \& Sarrias, 2010). Lo mismo ocurre cuando se consideran los fuertes encadenamientos comerciales que muchos de los sectores seleccionados tienen con empresas localizadas en la Región Metropolitana. En este sentido, no es raro que, en casos como la minería en el norte del país, la idea del cluster haya adquirido, implícita o explícitamente, un carácter más nacional (concentrador) que local. Esta 
idea es destacada por la OCDE (2009), cuando señala que actualmente los clusters "se concentran más en las estrategias nacionales que en las oportunidades regionales" (p. 113), y subraya las dificultades que este planteamiento provoca no solo para la consolidación de clusters arraigados en un territorio, sino también para el aprovechamiento de las diversas opciones de desarrollo que ofrecen las distintas regiones chilenas no metropolitanas.

\section{Conclusiones}

A pesar del crecimiento experimentado por Chile durante las últimas décadas, la fuerte primacía de la Región Metropolitana se mantiene con firmeza. El país ha superado, sin embargo, el nivel de producto per cápita que, según varias estimaciones econométricas internacionales, hace de la concentración un obstáculo para el crecimiento. Esta relación negativa no ha sido considerada todavía por las políticas nacionales orientadas a promover el crecimiento nacional. Las declaraciones públicas a favor de una mayor desconcentración siguen centradas en argumentos basados en la equidad espacial, lo cual, si bien es importante, reduce la magnitud del problema, un problema cuya gravedad se agudiza con la tendencia creciente de la desigualdad espacial experimentada durante las dos últimas décadas.

Varios son los factores que contribuyen a que la geografía económica de Chile se mantenga polarizada y no inicie el proceso de desconcentración que su nivel de desarrollo permitiría prever. En el ámbito del comportamiento de los mercados, la apertura comercial no ha debilitado los fuertes encadenamientos productivos de las regiones periféricas con el centro económico del país, con el que mantienen un alto déficit comercial. Este patrón de intercambio entre regiones hace que la Región Metropolitana capture una proporción significativa de los beneficios derivados de los proyectos de inversión realizados en otras regiones, incluidos los orientados a la promoción de clusters productivos. Un resultado parecido se desprende de la movilidad laboral, que, en contra de lo previsto por las teorías clásicas de la economía regional, no ha reducido las desigualdades regionales en salarios y empleo. En este punto destaca el efecto concentrador de la conmutación entre regiones o de larga distancia, favorecida por la regulación laboral sobre el trabajo por turnos.

Más llamativo aún resulta el carácter concentrador de muchas de las políticas públicas, incluso de aquellas cuyo objetivo declarado es la reducción de la desigualdad entre regiones, como el FNDR, cuya asignación ha sido, en los últimos tiempos, más favorable a la Región Metropolitana que al resto de las regiones. Además, políticas como la Estrategia Nacional para la Competitividad pierden su carácter territorial, basado en la formación de clusters regionales, mientras que la política monetaria sigue sustentada principalmente en datos de la Región Metropolitana y desconoce sus efectos heterogéneos en el espacio. Estos ejemplos dan cuenta de un marco institucional y político caracterizado por el "favoritismo", que promueve el exceso de concentración y genera incentivos económicos y políticos para que los recursos y los trabajadores más calificados se dirijan mayoritariamente a la Región Metropolitana. 
La reducción de la desigualdad espacial es un objetivo que debe incorporarse de manera expresa en el diseño de un nuevo modelo de crecimiento para Chile, y ello no solo por motivos de equidad, sino también para promover una mayor eficiencia en el país. En este punto, tal y como señalan Davis y Henderson (2003), la política pública y el marco institucional que de ella se deriva desempeñan una función clave en la reducción de las deseconomías de escala que se producen en el centro y en la posibilidad de aprovechar las oportunidades que ofrece la periferia. El avance hacia una menor concentración regional requiere, por un lado, de un verdadero proceso de descentralización administrativa que permita a todas las regiones tomar decisiones sobre el tipo de proyectos que les resultan más apropiados; y, por otro, de un conjunto de políticas nacionales que no sean ciegas a la dimensión espacial. Las palabras de Friedman y Stöhr (1966), hace más de cuarenta años, siguen siendo ciertas hoy y su puesta en práctica más urgente.

\section{Referencias bibliográficas}

Ades A. \& Glaeser, E. (1995). Trade and circuses. Explaining urban giants. Quarterly Journal of Economics, 110(1), 195-227.

Aroca, P. (2004). Migración interregional en Chile. Modelos y resultados 1987-2002. Revista del Centro Latinoamericano de Demografia (Celade): Notas de Población, 31(78), 97-154.

Aroca, P. (2009). Desigualdades regionales en Chile. Foreign Affairs Latinoamérica, 9(1), 53-63.

Aroca, P. \& Atienza, M. (2008). La conmutación regional en Chile y su impacto en la Región de Antofagasta. EURE, 34(102), 97-121. doi: 10.4067/S0250-71612008000200006.

Aroca, P. \& Hewings, G. J. D. (2002). Migration and labor market adjustment: Chile, 1977-1982 and 1987-1992. Annals of Regional Science, 36, 197-218.

Atienza M. (2006). Industrial agglomeration in Chilean regions. En P. Aroca \& G. Hewings (Eds.), Structure and structural change in the Chilean economy (pp. 199-224). Nueva York: Palgrave MacMillan.

Atienza, M., Lufín, M. \& Sarrias, M. (2010). División espacial del trabajo en Chile, 1992-2002. [Informe Anual 2009, Observatorio Regional de Desarrollo Humano]. Antofagasta: Ediciones Universitarias, Universidad Católica del Norte.

Banco Mundial (2009). World Development Report 2009. Reshaping Economic Geography. Washington, DC: Autor.

Benavente, D. (2007). Descentralización. La revolución olvidada. Temuco: Editorial Universidad Católica de Temuco.

Bravo, H. F., García C. J., Mies V. \& Tapia M. (2003). Heterogeneidad de la transmisión monetaria: efectos sectoriales y regionales. Documento de Trabajo, n. 235 , Banco Central de Chile.

Brülhart, M. \& Sbergami, F. (2009). Agglomeration and growth: Cross-country evidence. Journal of Urban Economics, 65(1), 48-63.

Carlin, A. (2009). Distribución espacial de la población y política comercial en Chile, 1865-2002. Seminario de Título de Pregrado, Instituto de Economía, Pontificia Universidad Católica de Chile. 
Combes, P., Lafourcade, M., Thisse, J. \& Toutain, J. (2008). The rise and fall of spatial inequalities in France: A long-run perspective. CEPR Discussion Paper, n. ${ }^{\circ} 7017$. Londres: Centre for Economic Policy Research. http://www.cepr.org/pubs/dps/DP7017.asp.

Davis, J. C. \& Henderson, J. V. (2003). Evidence on the political economy of the urbanization process. Journal of Urban Economics, 53(1), 98-125.

De Laire, F. (1999). La trama invisible o los claroscuros de la flexibilidad. Cuaderno de Investigación, n. ${ }^{\circ}$ 8. Santiago: Dirección del Trabajo, Chile.

Duranton, G. \& Puga, D. (2001). Nursery cities. Urban diversity, process innovation, and the life cycle of products. American Economic Review, 91(5), 1454-1477.

Duranton, G. (2009). Are cities engines of growth and prosperity for developing countries? En M. Spence, P. Clarke Annez \& R. M. Buckley (Eds.), Urbanization and growth (Cap. 3, pp. 67-114). Washington, DC: Banco Mundial.

Foxley, A. (1987). Chile y su futuro. Un país posible. Santiago de Chile: Corporación de Estudios para Latinoamérica (Cieplan).

Friedmann, J. \& Stöhr, W. (1966). The uses of regional science: Policy planning in Chile. Papers in Regional Science, 18(1), 207-222. doi:10.1111/j.1435-5597.1967.tb01366.x.

Fujita, M., Krugman, P. \& Venables, A. (1999). The spatial economy. Cities, regions and international trade. Cambridge, MA: The MIT Press.

Gaviria, A. \& Stein, E. (2000). The evolution of urban concentration around the world. A panel approach. Mimeo. Banco Interamericano de Desarrollo.

Geisse, G. \& Valdivia, M. (1978). Urbanización e industrialización en Chile. EURE, 5(15), 11-35.

Glaeser, E., Kallal, H., Scheinkman, J. \& Shleifer, A. (1992). Growth in cities. Journal of Political Economy, 100(6), 1126-1152.

Haddad, E. A. (1999). Regional inequality and structural changes. Lessons from the Brazilian experience. Aldershot, Brookfield: Ashgate.

Hanson, G. (1994). Regional adjustment to trade liberalization. NBER (The National Bureau of Economic Research, Cambridge, MA). Working Paper 4713. Publicado en Regional Science and Urban Economics, 28(4), 1 July 1998, 419-444.

Harris, C. (1954). The market as a factor in the localization of industry in the United States. Annals of the Association of American Geographers, 44(4), 315-348.

Henderson, J. V. (1999). The effect of urban concentration on economic growth. [Borrador]. Brown University, http://econ.pstc.brown.edu/faculty/henderson/.

Henderson, J. V. (2003). The urbanization process and economic growth: The so-what question. Journal of Economic Growth, 8(1), 47-71.

Henderson, J. V., Kunkoro, A. \& Turner, M. (1995). Industrial development in cities. Journal of Political Economy, 103(5), 1067-1090.

Hewings, G. (2009). Spatially blind trade and fiscal impact policies and their impact on regional economies. REAL Working Paper, University of Illinois, USA.

Hirschman, A. (1958). The strategy of economic development. New Haven: Yale University Press.

Instituto Nacional de Estadísticas (INE). (2009). Metodología del Índice de Precios al Consumidor (IPC); Base Diciembre de $2008=100$. Santiago de Chile: Autor.

Krugman, P. \& Livas, R. (1996). Trade policy and the Third World metropolis. Journal of Development Economics, 49(1), 137-150. 
Larraín, F. (1987). Desarrollo económico en democracia. Santiago: Ediciones Universidad Católica de Chile. Lufín, M. \& Atienza, M. (2010). Diferencias entre la composición sectorial y ocupacional de las principales ciudades chilenas. EURE, 36(108), 75-93. doi: 10.4067/S0250-71612010000200004.

Miranda Muñoz, C. (1997). Expansión urbana intercensal del Gran Santiago 1875-1992. Estadística y Economia (segundo semestre), 77-104.

Myrdal, G. (1957). Economic theory and under-developed regions. Londres: Duckworth.

Naciones Unidas (2008). World urbanization prospects. The 2007 revision. Nueva York: Autor.

Organización para la Cooperación y el Desarrollo Económicos (OCDE). (2009). Estudios territoriales de la OCDE. Chile. Santiago: Ministerio del Interior del Gobierno de Chile.

Paredes, D. (2011). Alternative theories for explaining the spatial wage inequality: a multilevel competition among human capital, NEG and amenities. Documento de trabajo IDEAR. Antofagasta: Universidad Católica del Norte, Facultad de Economía y Administración.

Puga, D. \& Venables, A. (1996). The spread of industry: spatial agglomeration in economic development. Journal of the Japanese and International Economies, 10(4), 440-464.

Puga, D. (1999). The rise and fall of regional inequalities. European Economic Review, 43(2), 303-334.

Richardson, H. (1987). The costs of urbanization. A four-country comparison. Economic Development and Cultural Change, 35(3), 561-580.

Rosen, K. \& Resnick, M. (1980). The size distribution of cities: an examination of the Pareto Law and primacy. Journal of Urban Economics, 8(2), 165-186.

Rosenthal, S \& Strange W. C. (2003). Geography, industrial organization, and agglomeration. Review of Economics and Statistics, 85(2), 377-393.

Sarrias, M. \& Aroca, P. (2011). Regional Business Cycle Synchronization in Chile. Documento de trabajo IDEAR. Antofagasta: Universidad Católica del Norte, Facultad de Economía y Administración.

Storey, K. (2010). Fly-in/Fly-out. Implications for community sustainability. Sustainability, 2(5), 1161-1181.

Universidad de Los Lagos (2010). Informe Final: Estudio articulación de actores para la descentralización. Santiago: Subsecretaría de Desarrollo Regional (Subdere), Gobierno de Chile.

Van Marrewijk, CH. (2005). General geographical economics model with congestion. Tinbergen Institute Discussion Paper, n. ${ }^{\circ}$ 05-100/2.

Waissbluth, M., con la colaboración de Inostroza, J. (2006). La reforma del Estado de Chile 1990 2005. De la confrontación al consenso. PUBLIC. Boletín Electrónico del Instituto de Gobernanza y Dirección Pública (IGDP) de ESADE (Escuela Superior de Administración y Dirección de Empresas, Barcelona), marzo. (www.esade.edu/public).

Wheaton, W. \& Shishido, H. (1981). Urban concentration, agglomeration economies, and the level of economic development. Economic Development and Cultural Change, 30(1), 17-30.

Williamson, J. (1965). Regional inequality and the process of national development: A description of the patterns. Economic Development and Cultural Change, 13(4), 3-45. 